\title{
Mitochondrial dysfunction defines a population of young people with type 1 diabetes at risk of kidney disease
}

\begin{abstract}
JM Forbes ${ }^{1}$, S Leung1, NB Flemming ${ }^{1}$, DA McCarthy¹, K Boot ${ }^{2}$, N De Silva ${ }^{3}$, LA Gallo ${ }^{1}$, D Johnson ${ }^{4}$, T Jones $^{5}$, J Couper ${ }^{6}$, K Donaghue ${ }^{7}$, MP Hodson ${ }^{8}$, T O'Moore-Sullivan ${ }^{3}$

${ }^{1}$ Mater Research Institute - The University of Queensland, TRI, Brisbane, Queensland; ${ }^{2}$ David Serisier Respiratory Biobank and ${ }^{3}$ Diabetes and Endocrine Centre, Mater Health Service, Brisbane, Qld; ${ }^{4}$ Metro South Health, Brisbane, Qld; ${ }^{5}$ Telethon Kid's Institute, Perth, WA; ${ }^{6}$ Women's and Children's Hospital, Adelaide, SA; ${ }^{7}$ Children's Hospital at Westmead, Sydney, NSW; ${ }^{8}$ Metabolomics Australia, The University of Queensland, St Lucia, Queensland; AUSTRALIA.
\end{abstract}

\section{Background/Rationale}

There is increasing evidence that kidney disease in Type 1 diabetes (T1D) begins during adolescence [1].

Elevations in urinary albumin creatinine ratio (UACR) within a normal range may predict those individuals at greater risk of developing microalbuminuria and an adverse profile of cardiovascular risk factors [2]. Mitochondrial dysfunction is appreciated as an early pathological mediator of diabetic kidney disease [3].

\section{Objective}

The objective of this study was to examine the relationships among mitochondrial function, UACR and estimated glomerular filtration rate (eGFR) in young people with T1D.

\section{Methods}

Inclusion criteria: Adolescents and young adults with previously diagnosed Type 1 diabetes were recruited. Exclusion criteria: Individuals with uncontrolled diabetes (defined as $\mathrm{HbA}_{1 c}>9.5$ or recurrent ketoacidosis), autoimmune diseases resulting in skeletal muscle wasting (such as multiple sclerosis), catabolic coeliac disease, Addison's disease, any congenital mitochondrial disease, previous $\mathrm{MI} /$ stroke, severe familial hypercholesterolaemia, pre-existing renal disease, pregnancy, or an inability to consent were excluded from the study. Research Design and Methods: Mean UACR was determined from 3 morning urine samples. The study population was divided into UACR tertiles (Table 1). SeaHorse $\mathrm{XF}$ analyser was used for mitochondrial functional assays in circulating leukocytes. Urinary metabolomics by LC MSMS, GCMS and HPLC measured central carbon metabolites, nucleotides, fatty and amino acids.

\begin{tabular}{|c|c|c|c|}
\hline uACR range ( $\mu \mathrm{g} / \mathrm{mmol})$ & $\begin{array}{l}\text { Lower Risk } \\
(\leq 0.66)\end{array}$ & $\begin{array}{l}\text { Middle Risk } \\
\text { (0.67-1.16) }\end{array}$ & $\begin{array}{l}\text { High Risk- Upper } \\
\quad(\geq 1.17)\end{array}$ \\
\hline $\mathbf{N}$ & 33 & 33 & 34 \\
\hline Sex (n Male, \%) & $(21,63.6 \%)$ & $(16,48.5 \%)$ & $(17,50 \%)$ \\
\hline Age (yr) & $21(17.5-22.5)$ & $20(17.5-22.0)$ & $19(17-23.25)$ \\
\hline Age at diagnosis (yr) & 11 (7-14.5) & $10(4-12.5)$ & $9.5(5.5-12.25)$ \\
\hline Diabetes duration (yr) & $9.879 \pm 4.904$ & $11.42 \pm 4.944$ & $10.65 \pm 5.762$ \\
\hline $\mathrm{HbA}_{1} \mathrm{C}(\%)$ & $8.0(7.3-8.55)$ & $8.2(7.45-8.7)$ & $8.45(7.975-9.1) *$ \\
\hline 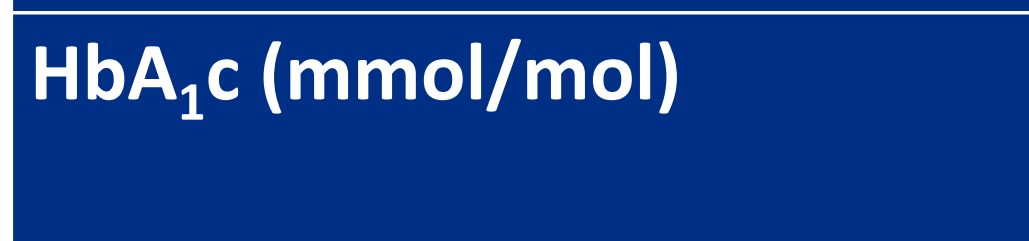 & $63.94(56.29-69.95)$ & $66.13(57.93-71.59)$ & $\begin{array}{c}68.86(63.67-75.96) \\
*\end{array}$ \\
\hline Fed BG (mmol/L) & $11.65(7.225-14.88)$ & $12(7.925-15.20)$ & $12.55(8.2-15.08)$ \\
\hline Height (m) & $1.75(1.665-1.795)$ & $1.73(1.675-1.785)$ & $1.71(1.65-1.82)$ \\
\hline Weight (kg) & $80(69-87)$ & $74(68.2-78.5)$ & $74.5(65.85-84.65)$ \\
\hline BMI $\left(\mathrm{kg} / \mathrm{m}^{2}\right)$ & $26(23-29.5)$ & $24(22.5-26.5)$ & $24.5(20.75-28.25)$ \\
\hline BMI (percentile) & $85(59-96)$ & $77(33-87)$ & $71(38-91)$ \\
\hline Mean uACR ( $\mu \mathrm{g} / \mathrm{mmol})$ & $0.5(0.415-0.57)$ & $0.82(0.73-0.93) *$ & $\begin{array}{c}1.73(1.483-4.425) \\
*+\end{array}$ \\
\hline $\mathrm{eGFR}_{\mathrm{CKD}-\mathrm{FP} \mathrm{I}}\left(\mathrm{ml} / \mathrm{min} / 1.73 \mathrm{~m}^{2}\right)$ & $134.7 \pm 9.5$ & $136.3 \pm 8.1$ & $137.8 \pm 11.4^{*}$ \\
\hline $\begin{array}{l}\text { eGFR }_{\text {ScHWARTZ }} \\
\left(\mathrm{m} / \mathrm{min} / 1.73 \mathrm{~m}^{2}\right)\end{array}$ & $108(97.5-121.8)$ & $117(107.5-124.5)$ & 119 (104-130.5)* \\
\hline
\end{tabular}

eGFR and UACR Relationships
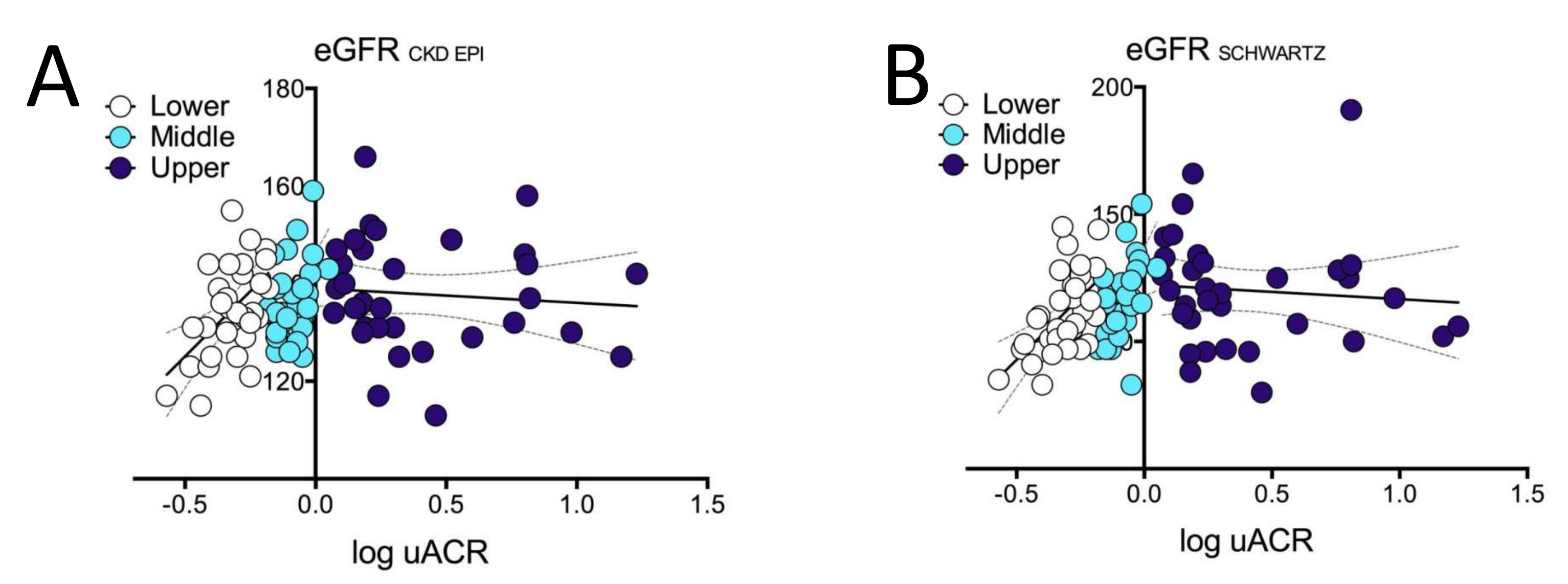

Figure 1: Generalised linear modelling of eGFR and log UACR. A majority (99.7\%) of participants had hyperfiltration [eGFR ${ }_{\text {KKD-EPI }} ; 135.0$ (13.8) $\mathrm{ml}^{-\mathrm{min}^{-}}$ $\left.{ }^{1} \cdot \mathrm{m}^{-2}\right)$. UACR and eGFR relationships are shown corrected for age, sex, diabetes duration and $\mathrm{HbA}_{1 \mathrm{C}}$. A) eGFR CKD EPI and log UACR, Lower; $r=+0.52, P=0.006$; Upper (high) risk tertile; $r=-0.25, P=0.49$. B) eGFR sCHWARTz and log UACR, Lower; $r=+0.58, P=0.002$; Upper (high) risk tertile; $r=-0.28, P=0.12$.

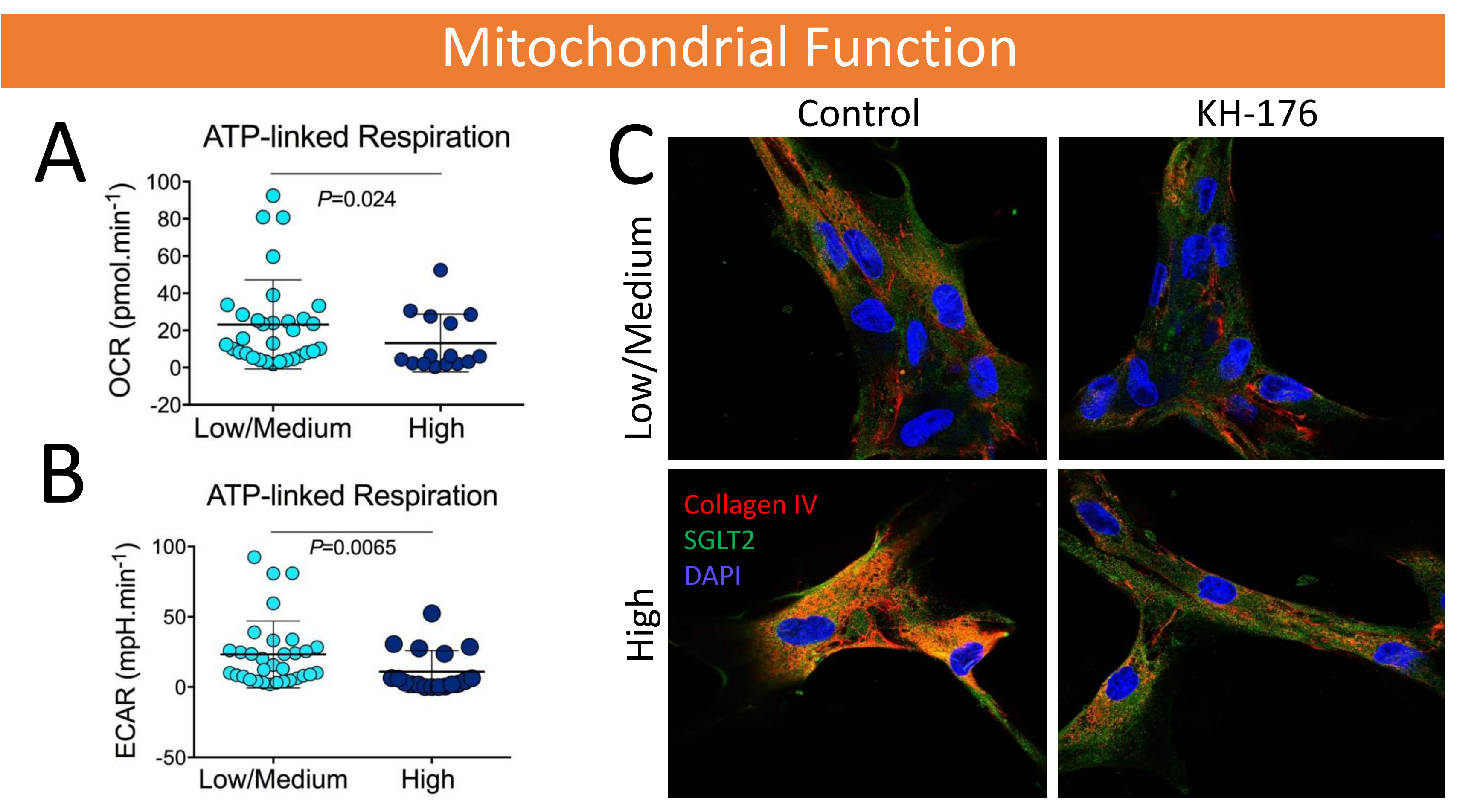

Figure 2: A) Oxygen consumption rate (OCR) and B) Extracellular acidification rate (ECAR) in leukocytes from individuals with T1D ( $N=52)$. C) Healthy primary human proximal tubule cells exposed to plasma (4\%) from age, sex and diabetes duration matched individuals with T1D. KH176 - therapy targeting mitochondrial function (Khondrion).

\section{Urinary Metabolomics}

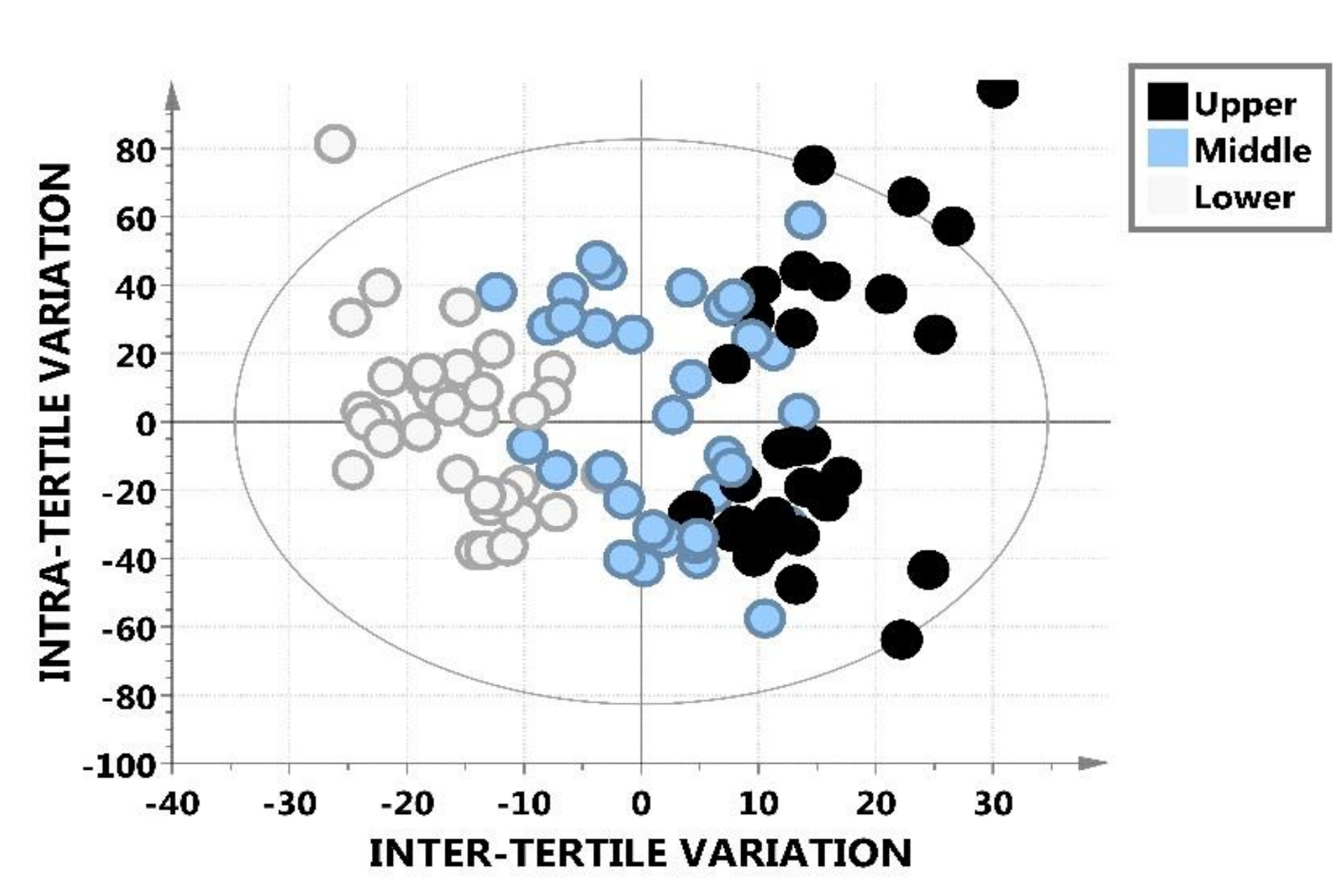

Figure 3: Multivariate model scores plot of urinary metabolites from T1D individuals, demonstrating separation of tertiles along the predictive component axis $(x)$ axis, with intra-tertile variability along the orthogonal (y) axis. 12 metabolites separated the high risk tertile.

\section{Conclusions}

Young individuals with type 1 diabetes in the high risk tertile of UACR have increased eGFR associated with a profile of mitochondrial dysfunction. Refs: [1] PMID:24198300 [2] PMID:17257274; 11128358 [3] PMID:26831938.
Table 1: General clinical characteristics and biochemical parameters of study cohort. Data are shown as mean $\pm \mathrm{SD}, \mathrm{n}(\%)$, or median (interquartile range). ${ }^{*} P<0.05$ vs Lower Tertile; $+P<0.05$ vs Middle Tertile by one-way ANOVA and Tukey's post hoc or Kruskal Wallis with Dunn's post hoc testing. AParticipants under 18 years of age only. BG, blood glucose concentrations; BMI, body mass index; eGFR, estimated glomerular filtration rate; $\mathrm{HbA}_{1 \mathrm{c}}$, glycated haemoglobin. Using tertiles of $\mathrm{HbA}_{1 \mathrm{C}}$ no stratification of $\mathrm{UACR}$ or eGFR is evident using generalized linear modelling.
Josephine.forbes@mater.uq.edu.au NIDDK Pilot Award

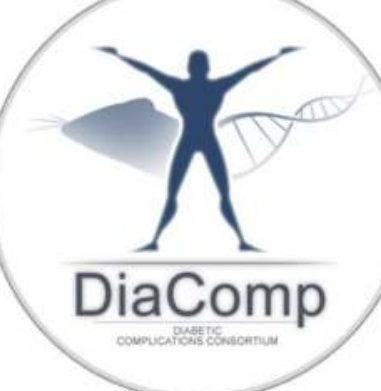

